\title{
DISCOVERY OF LUMINOUS STAR FORMATION IN PMN 1452-5910/IRAS 14482-5857: THE PTERODACTYL NEBULA
}

\author{
D. I. JONES ${ }^{1}$ AND C. R. BRAIDING ${ }^{2}$ \\ ${ }^{1}$ Department of Astrophysics/IMAPP, Radboud University, Heijendaalseweg 135, 6525 AJ Nijmegen, The Netherlands; d.jones@astro.ru.nl \\ ${ }^{2}$ School of Physics, University of New South Wales, 2052, Sydney, Australia \\ Received 2014 April 16; accepted 2014 November 25; published 2015 January 22
}

\begin{abstract}
We present sensitive 1-3 GHz ATCA radio continuum observations of the hitherto unresolved star-forming region known as either IRAS 14482-5857 or PMN 1452-5910. At radio continuum frequencies, this source is characterized by a "filled bubble" structure reminiscent of a classical Hit region, dominated by three point sources and surrounded by low surface brightness emission out to the $\sim 3^{\prime} \times 4^{\prime}$ source extent observed at other frequencies in the literature. The infrared emission corresponds well to the radio emission, with polycyclic aromatic hydrocarbon emission surrounding regions of hot dust toward the radio bubbles. A bright $4.5 \mu \mathrm{m}$ point source is seen toward the center of the radio source, suggesting a young stellar object. There is also a linear, outflowlike structure radiating brightly at 8 and $24 \mu \mathrm{m}$ toward the brightest peak of the radio continuum. In order to estimate the distance to this source, we have used Mopra Southern Galactic Plane CO Survey ${ }^{12} \mathrm{CO}(1-0)$ and ${ }^{13} \mathrm{CO}(1-0)$ molecular line emission data. Integrated intensity, velocity at peak intensity, and line fitting of the spectra all point toward the peak centered at $V_{\mathrm{LSR}}=-1.1 \mathrm{~km} \mathrm{~s}^{-1}$ being connected to this cloud. This infers a distance to this cloud of $\sim 12.7 \mathrm{kpc}$. Assuming this distance, we estimate a column density and mass toward IRAS 14482-5857 of $\sim 1.5 \times 10^{21} \mathrm{~cm}^{-2}$ and $2 \times 10^{4} M_{\odot}$, implying that this source is a site of massive star formation. Reinforcing this conclusion, our broadband spectral fitting infers dust temperatures of 19 and $110 \mathrm{~K}$, emission measures for the subparsec radio point source of EM $\sim 10^{6-7} \mathrm{pc} \mathrm{cm}^{-6}$, electron densities of $n_{\mathrm{e}} \sim 10^{3} \mathrm{~cm}^{-3}$, and photon ionization rates of $N_{\mathrm{Ly}} \sim 10^{46-48} \mathrm{~s}^{-1}$. The evidence strongly suggests that IRAS 14482-5857 is a distant-hence intense-site of massive star formation.
\end{abstract}

Key words: ISM: clouds - HII regions - ISM: individual objects (IRAS14482-5857, PMN 1452-5910)

\section{INTRODUCTION}

Massive stars are the principle source of heavy elements in the universe; they disperse material and modify their environment through winds, massive outflows, expanding HII regions, and supernovae (Zinnecker \& Yorke 2007). This creates an important source of mixing and turbulence for the interstellar medium (ISM) of galaxies (Zinnecker \& Yorke 2007), making massive star formation and a theory for it hugely important for modern astrophysics. For all the effect that massive stars have on their host galaxies, we understand little about the conditions of their formation, especially in the earliest phases. This is because while a fully developed massive star or star cluster can be observed in the optical as well as at other wavelengths, a high amount of dust extinction during formation makes this a demanding proposition. Coupled with the fact that they are rare and possess short formation timescales, this makes observing the earliest phases of massive star formation in large numbers fiendishly difficult (Zinnecker \& Yorke 2007).

Sites of massive star formation have traditionally been discovered using large surveys in the infrared, such as by using infrared colors (Molinari et al. 1996; Walsh et al. 1997), dust emission (Hill et al. 2005), or molecular line emission (Walsh et al. 2011; Burton et al. 2013). However, extending the distance to which sites of massive star formation in the Galaxy can be observed is necessarily difficult due to the large regions that must be observed to a higher sensitivity. This is summarized by the statistic given by large surveys of mass as a function of distance that can be observed. For instance, the $\mathrm{H}_{2} \mathrm{O}$ Galactic Plane Survey (HOPS) can detect a $20 \mathrm{~K}, 400 M_{\odot}$ cloud at the $5 \sigma$ level at $3.2 \mathrm{kpc}$ and a $3 \times 10^{4} M_{\odot}$ clouds anywhere in the Galaxy (Walsh et al. 2011). Given this, it is becoming imperative to use the ever growing number of highquality surveys across many wavebands to characterize starforming regions in a more systematic and comprehensive manner so as to increase the number of known massive starforming regions.

Here we report serendipitous radio continuum observations at $1-3 \mathrm{GHz}$ as well as an examination of the archival data toward what may be a hitherto unknown site of massive star formation; IRAS 14482-5857. A search of SIMBAD ${ }^{3}$ within a radius of 2 arcmin revealed only survey information for the radio (cataloged as Parkes-MIT-NRAO (PMN) 1452-5910) and infrared source (cataloged) as IRAS 14482-5857, but nothing else. ${ }^{4}$ The PMN 1452-5910 and IRAS 14482-5857 sources are positionally coincident, and hereafter, we refer to this source simply as IRAS 14482-5857. Figure 1 shows IRAS 14482-5857, and its surrounding environment at $843 \mathrm{MHz}$ from the Molongolo Observatory Synthesis (MOST; Bock et al. 1999) Galactic Plane Survey (MGPS; Mauch \& Murphy 2003). IRAS 14482-5857 lies near the large $\left(40^{\prime} \times 35^{\prime}\right)$ supernova remnant, G318.2 + 0.1, along the Galactic plane at a position of $\alpha=14^{\mathrm{h}} 52^{\mathrm{m}} 05^{\mathrm{s}} 3$, $\delta=-59^{\circ} 10^{\prime} 08$ " 58 (J2000.0).

\footnotetext{
3 http://simbad.u-strasbg.fr/simbad/

4 SIMBAD cites an X-ray study (Bocchino et al. 2001) in reference to PMN 1452-5910, which only contains the source IRAS 14498-5856. This reference is spurious.
} 


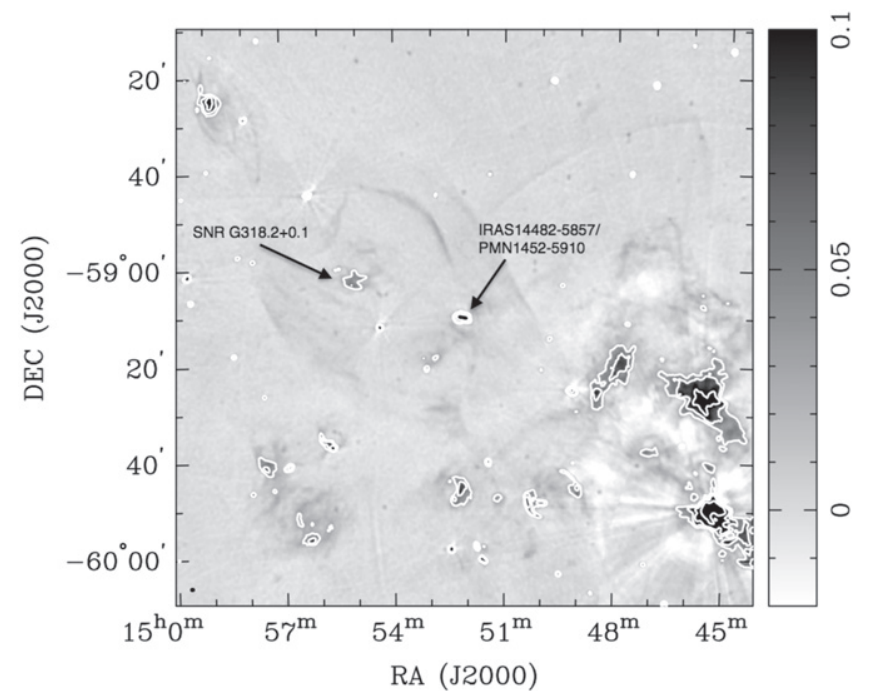

Figure 1. $843 \mathrm{MHz}$ total intensity image of the region surrounding IRAS 14482-5857, overlaid with the same (white) contours at $30(=5 \sigma), 60,120$, 240 , and $480 \mathrm{mJy} /$ beam. The beam at this declination is $43^{\prime \prime} \times 50^{\prime \prime}$, and is shown in the lower left-hand corner.

\section{NEW OBSERVATIONS AND ARCHIVAL DATA}

In this section we describe the new data on IRAS 14482-5857 that was obtained using ATCA as well as the archival data obtained during a literature search and the survey data that we possessed.

\subsection{New Australia Telescope Compact Array Data}

The ATCA data presented here are a part of an observational campaign toward a nearby object, but IRAS 14482-5857 is contained within the field of view at $1-3 \mathrm{GHz}$. The observations and data reduction technique will be detailed elsewhere, but we give a brief overview here.

A (hexagonal) mosaic observation of the region was performed with the ATCA on 2013 May 5 and 2013 September 6 in the $6.0 \mathrm{C}$ and 1.5A array configurations, respectively. Both array configurations have a minimum baseline of $153 \mathrm{~m}$ up to a maximum of $6000 \mathrm{~m}$ (for the $6.0 \mathrm{C}$ configuration only-the maximum 1.5A baseline is $4469 \mathrm{~m}$ ). The observations used the $16 \mathrm{~cm}$ receiver with the CFB $1 \mathrm{M}-0.5 \mathrm{k}$ correlator, which gives a $2 \mathrm{GHz}$ bandwidth with 2048 channels of $1 \mathrm{MHz}$ width over the 1-3 GHz band. We split up the band into four $512 \mathrm{MHz}$ wide sub-bands, and use only the 1332,1844 , and $2868 \mathrm{MHz}$ bands here. Standard calibration and imaging methods were used within the MIRIAD software reduction package. The flux density calibrator used was PKS B1934-638, bootstrapped to a flux density of $11.986 \pm 0.005 \mathrm{Jy} / \mathrm{beam}$ at $2100 \mathrm{MHz}$. These bands

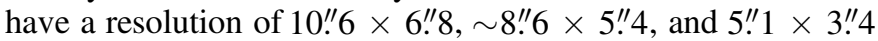
and $1 \sigma \mathrm{rms}$ sensitivities of $\sim 400, \sim 200$, and $\sim 100 \mu \mathrm{Jy} / \mathrm{beam}$ at 1332,1844 , and $2868 \mathrm{MHz}$, respectively. We found a systematic uncertainty of $\sim 5 \%$ for the observations and all errors quoted in this paper use this added in quadrature to the local rms sensitivity. It is important to note that these observations will recover spatial information only up to $\sim 5.5$, $\sim 4^{\prime}$, and $\sim 2$ '7 at 1332,1844 , and $2868 \mathrm{MHz}$. Given that the source size is $\sim 3^{\prime} \times 4^{\prime}$, the upper band may not fully recover the entire flux density.

\subsection{Archival Data}

In order to better characterize the IRAS 14482-5857 source, we have performed a literature search and have obtained data from several surveys. Here we describe the data products we have obtained and used.

\subsubsection{Millimeter and Infrared Data}

Since this source is identified in SIMBAD as IRAS 144825857, we know that it is detected at infrared frequencies. Hence, we have obtained mid-infrared data from IRAS at 12, 25, 60, and $100 \mu \mathrm{m}$. We have also obtained IRAC Mid-course Space eXperiment (MSX) and Spitzer Galactic Legacy Infrared Mid-Plane Survey Extraordinaire (GLIMPSE) data at the shorter wavelengths of $8.28,12.13,14.65$, and $21.34 \mu \mathrm{m}$ for the A, C, D, and E bands of $M S X$ and at 3.6, 4.5, 5.8, and $8 \mu \mathrm{m}$ for the GLIMPSE bands. Emission at $3.6 \mu \mathrm{m}$ is dominated by stellar emission and is usually represented in three-color (i.e., false-color) images as blue. Bright emission at $4.5 \mu \mathrm{m}$ is thought to be due to shocks arising from outflows from protostellar objects interacting with the ambient ISM (Cyganowski et al. 2008). 5.8 and $8.0 \mu \mathrm{m}$ emission is thought to be due to polycyclic aromatic hydrocarbons (PAHs), which are particularly useful for highlighting dust emission since they are very sensitive to infrared emission (Churchwell et al. 2009). We have also made use of the longer wavelength MIPSGAL $24 \mu \mathrm{m}$ emission, which also traces dust emission. Finally, we have searched archival papers for information on dust clumps observed at $1.1 \mathrm{~mm}(150 \mathrm{GHz})$, which is also thought to be a powerful discriminator of the evolutionary stage of massive star formation (see, for instance, Breen et al. 2010b).

\subsubsection{Mopra ${ }^{12} \mathrm{CO}(1-0)$ and ${ }^{13} \mathrm{CO}(1-0)$ Data}

In order to establish the presence (or absence) of molecular gas toward IRAS 14482-5857, we have obtained molecular line emission data from the Mopra telescope from the Mopra Southern Galactic Plane CO Survey (Burton et al. 2013). This survey used the Mopra telescope, which is a single $22 \mathrm{~m}$ dish radio telescope located $\sim 450 \mathrm{~km}$ northwest of Sydney, Australia $\left(31^{\circ} 16^{\prime} 04^{\prime \prime} \mathrm{S}, 149^{\circ} 05^{\prime} 59^{\prime \prime} \mathrm{E}, 866 \mathrm{~m}\right.$ a.s.l. $)$ to survey a region of the southern Galactic plane from $l=305^{\circ}-345^{\circ}$ and $b= \pm 0.5$. The survey observations utilized the UNSW Mopra wide-band spectrometer (MOPS) in zoom mode with the $3 \mathrm{~mm}$ receiver, which operates in a frequency range of $77-117 \mathrm{GHz}$, and for the relevant observations here, the $8 \mathrm{GHz}$ wide bandwidth was centered on $112.5 \mathrm{GHz}$. The fast-mapping zoom mode of MOPS allows observations in up to 8 windows simultaneously, where each window is $137.5 \mathrm{MHz}$ wide and contains 4096 channels in each of 2 polarizations. This observational set-up yields a spectral resolution of $\sim 0.09 \mathrm{~km} \mathrm{~s}^{-1}$ over a velocity range of $\sim-500$ to $500 \mathrm{~km} \mathrm{~s}^{-1}$ depending on the line sampled and a spatial resolution of $\sim 35^{\prime \prime}$, with a main beam efficiency of 0.55 at $115 \mathrm{GHz}$. The observations were conducted using the "fast on-the-fly" (FOTF) method and reduced as per the methods described in (Burton et al. 2013). This resulted in a $1 \sigma$ sensitivity for the ${ }^{12} \mathrm{CO}$ and ${ }^{13} \mathrm{CO}$ (hereafter simply ${ }^{12} \mathrm{CO}$ and ${ }^{13} \mathrm{CO}$ ) lines of $\sim 1.5 \mathrm{~K}$ and $\sim 0.7 \mathrm{~K}$ per $0.1 \mathrm{~km} \mathrm{~s}^{-1}$ velocity channel, respectively. 

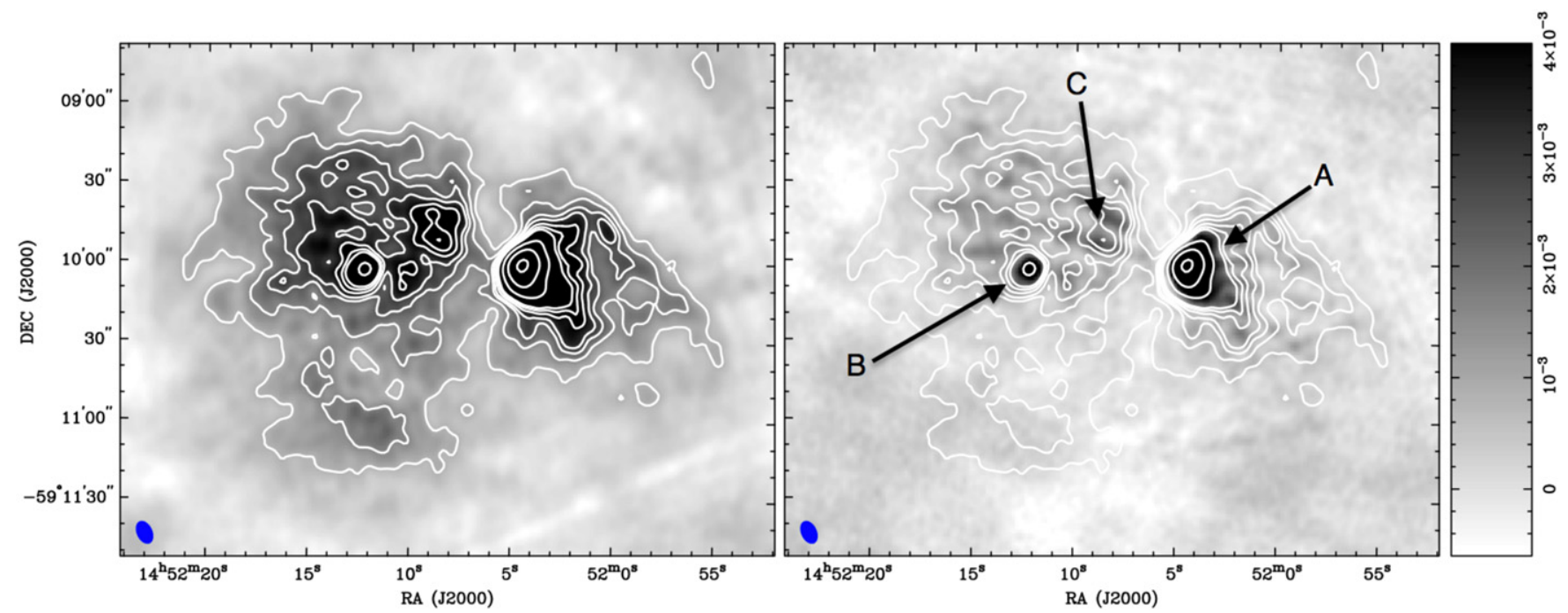

Figure 2. Total intensity images of our ATCA data at 1844 (left) and 2868 (right) of IRAS 14482-5857. The contours in both frames are $1844 \mathrm{MHz}$ contours at $0.8,1$, $2,4,6,7,8,10,20,40$, and $80 \mathrm{mJy} / \mathrm{beam}$. The intensity scale is linear and runs from -0.6 to $4 \mathrm{mJy} / \mathrm{beam}$. At $1844 \mathrm{MHz}$, the rms noise is $\sim 200 \mu \mathrm{Jy} / \mathrm{beam}$ (so that $0.8 \mathrm{mJy} / \mathrm{beam}=4 \sigma)$, whereas at $2868 \mathrm{MHz}$, the rms noise is $\sim 100 \mu \mathrm{Jy} / \mathrm{beam}$. The beams are 8"'6 $\times 55^{\prime \prime} 4$ and a position angle of $25^{\circ} .1$ and 5 ". $1 \times 33^{\prime \prime} 4$ at a position angle of 21.6 at 1844 and $2868 \mathrm{MHz}$ respectively. The beam are shown in the lower left-hand corner of both images. The regions that are peaks in intensity are labeled "A," "B," and "C" in the right-hand panel.

\subsection{3. $\mathrm{NH}_{3}(1,1)$ and $(2,2)$ Inversion \\ Transition and $\mathrm{H}_{2} \mathrm{O}$ Maser Emission}

As presaged above, it is thought that massive stars are formed in dense clumps of cold gas. $\mathrm{CO}$ is known to be a particularly poor tracer of cold, dense molecular material because it "freezes out" in temperatures of 10s of K (Nicholas et al. 2011) and suffers optical depth problems at moderate densities. Thus, we have obtained data from the HOPS (Walsh et al. 2011).

HOPS is a survey of the $\sim 100^{\circ}$ region of the Galactic plane (from $-70^{\circ}>l>30^{\circ},|b|<0.5$ ) using the Mopra telescope in the $12 \mathrm{~mm}$ waveband in on-the-fly mode also using MOPS. HOPS is particularly useful for tracing massive star formation because in a single survey it traces the $23 \mathrm{GHz}$ water maser transition that is known to trace star formation as well as the versatile inversion transitions of the ammonia molecule (which do not suffer from freeze-out and trace cold, dense clumps). The $\mathrm{NH}_{3}(1,1)$ and $(2,2)$ data have a resolution of $2^{\prime}$ over velocities ranging from $\pm 200 \mathrm{~km} \mathrm{~s}^{-1}$. The median sensitivity of the $\mathrm{NH}_{3}$ data cubes is $\sigma_{T_{\mathrm{mb}}}=0.20 \pm 0.06 \mathrm{~K}$.

\section{RESULTS}

\subsection{Radio Continuum Emission}

Figure 2 shows total intensity radio continuum images of IRAS 14482-5857 at 1844 and $2868 \mathrm{MHz}$. The source exhibits a bi-lobal structure, consisting of diffuse, low surface brightness emission as well as at least three strong peaks with a single peak seen in the western lobe of the bubbles and two in the eastern one. The lack of diffuse emission at $2868 \mathrm{MHz}$ in Figure 2 (right) shows the results of the spatial filtering of interferometers. However, we suggest that the difference between the true flux density distribution of the source and that shown here is not significant. This is because, as is shown in Table 1, the integrated flux densities of the strong point sources are consistent with optically thin thermal emission and match those seen at lower $(843 \mathrm{MHz})$ and higher $(4500 \mathrm{MHz})$ frequencies. Spectral index maps (see below) also show optically thin thermal emission over most of the bubbles, suggesting that significant amounts of flux at $2868 \mathrm{MHz}$ have not been filtered. At $1332 \mathrm{MHz}$, as Figure 3 shows, the opposite is true. This figure shows IRAS 14482-5857 at $1332 \mathrm{MHz}$, and illustrates that there is ample diffuse emission at this frequency since it samples structures larger than the source itself. Indeed, the larger source extent, along with an integrated flux density for the entire source of $\sim 2$ Jy suggests that at this frequency, a significant amount of background emission from the Galactic plane is being sampled. Hence we do not use this datum in fitting the centimeter radio continuum emission. Again-although in the opposite sense-the flux density reported from the PMN survey at $4500 \mathrm{MHz}$ (using single dishes) is commensurate with the 1844 and $2868 \mathrm{MHz}$ flux densities shown in Table 1 . Thus we conclude that the additional flux density in the $1332 \mathrm{MHz}$ is background emission rather than reflecting additional flux density from the source itself.

Figure 4 shows a spectral index map of IRAS 14482-5857 between 1844 and $2868 \mathrm{MHz}$. We define the spectral index $\alpha$ between two frequencies $\nu$ as $\alpha \equiv d \log S_{\nu} / d \log \nu$. Figure 4 shows the spectral index variations between -1 (indicative of non-thermal emission, e.g., synchrotron emission) and +2 (indicative of thermal emission, e.g., optically thick thermal emission). This indeed illustrates the point made above that IRAS 14482-5857 consists of optically thin thermal emission interspersed with some non-thermal emission toward the edge of the radio bubbles, which is most likely due to low signal-tonoise in the $2868 \mathrm{MHz}$ data. The same map between 1332 and $1844 \mathrm{MHz}$ shows a spectro-morphological structure similar to that shown in Figure 4, but with steeper spectral indices, suggestive of contaminating flux density.

We have characterized the strong peaks observed at 1332, 1844 , and $2868 \mathrm{MHz}$, and labelled as sources A, B, and C in the right-hand panel of Figure 2. This was done by fitting Gaussian sources to the data using the MIRIAD task imsad without fitting a background to subtract. We do not subtract any background emission because, as shown above, the size 


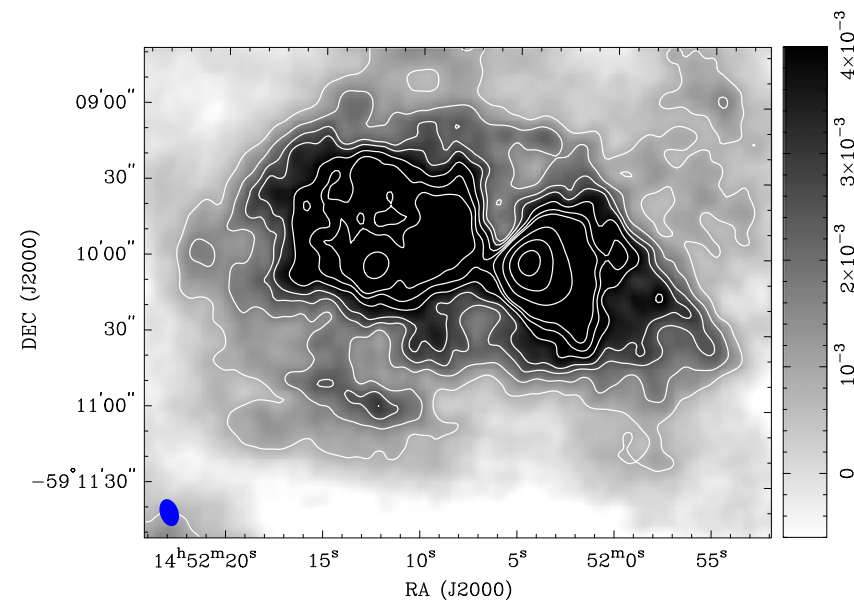

Figure 3. Total intensity image of IRAS $14482-5857$ at $1332 \mathrm{MHz}$. The (white) contours are at levels of $0.2,0.4,0.8,1,2,4,8,16,32$, and $64 \mathrm{mJy} / \mathrm{beam}$. The intensity scale is linear and runs from -2 to $6 \mathrm{mJy} / \mathrm{beam}$. The rms noise is $\sim 400 \mu \mathrm{Jy} / \mathrm{beam}$ (first contour is $0.8 \mathrm{mJy} / \mathrm{beam} \sim 2 \sigma$ ). The beam is $10 . \prime 6 \times 6 . " 8$ and a position angle of $18^{\circ} .8$ and is shown in the lower left-hand corner.

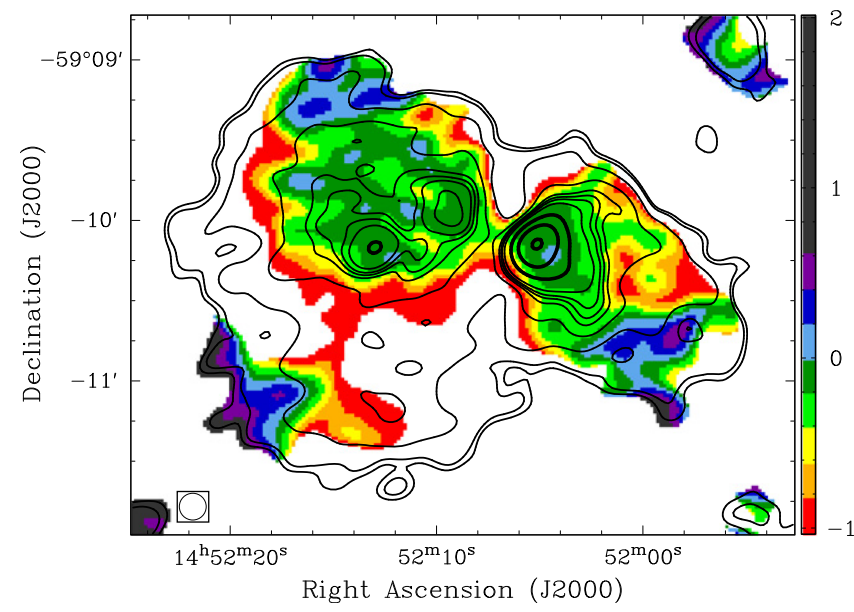

Figure 4. Spectral index map of IRAS 14482-5857 between 1844 and $2868 \mathrm{MHz}$ at a common resolution of $10^{\prime \prime} \times 10^{\prime \prime}$. The (black) contours are $1844 \mathrm{MHz}$ emission at $0.8,1,2,3,4,5,6,7,8,12$, and $16 \mathrm{mJy} / \mathrm{beam}$. This illustrates the non-thermal nature of parts of the radio bubbles. The peaks of intensity within the radio bubbles show a thermal structure, commensurate with the total integrated flux.
$1332 \mathrm{MHz}$, however. Table 1 shows the results of this fitting and shows the source name (column 1), frequency fitted (column 2), best-fit positions (columns 3 and 4), angular sizes, and position angles (column 5) of the best-fit Gaussians as well as the peak and integrated flux density (columns 6 and 7). As can be seen in Figure 2 (right), the second peak in the eastern bubble is not detected at $2868 \mathrm{MHz}$. The diffuse flux density has average values of 6,3 , and $3 \mathrm{mJy} / \mathrm{beam}$ at 1332,1844 , and $2868 \mathrm{MHz}$ respectively, with the stronger average flux density at $1332 \mathrm{MHz}$ probably due to the background flux density at scales larger than the source. These levels were used as a cutoff level for the modeling of the bright radio peaks.

\subsection{Archival Data}

\subsubsection{Radio Continuum and Millimeter Data}

Data associated with PMN 1452-5910 comes from the PMN (Wright et al. 1994) and comprises a flux density and angular extent of $1.14 \pm 0.06 \mathrm{Jy}$ and $3^{\prime} \times 4^{\prime}$ at a position angle of 128.5 , respectively.

A SIMBAD search of IRAS 14482-5857 contains flux densities from a coincident source found using the QUaD telescope from their Galactic plane survey (Culverhouse et al. 2010), which lists a flux density of $2.35 \pm 0.44$ and $1.90 \pm 0.3 \mathrm{Jy}$ at 100 and $150 \mathrm{GHz}$, respectively (Culverhouse 2011). This flux density comes from a source extent of $3.9 \times 3.1$ and $2 ! .8 \times 1.7$ at 100 and $150 \mathrm{GHz}$, respectively, roughly matching that found at centimeter wavelengths (i.e., the PMN survey and the extent of the source in Figure 1). Culverhouse (2011) also characterize this source as a UCHII region based on the Wood-Churchwell criteria which is based on the IR colors (Wood \& Churchwell 1989).

\subsubsection{Mopra CO(1-0) Molecular Line Emission}

Figure 5 shows an integrated-intensity (i.e., mom 0 ) image for the ${ }^{12} \mathrm{CO}$ data integrated over the velocity range of -10 to $+10 \mathrm{~km} \mathrm{~s}^{-1}$. This velocity-range is motivated by inspection of velocity-at-peak-intensity images (not shown here) derived for this region that show that the peak of emission within the (white) contours of Figure 5, as well as the more extended emission, lie between this velocity range. Integrating the ${ }^{12} \mathrm{CO}$ emission between -55 and $-35 \mathrm{~km} \mathrm{~s}^{-1}$ (images also not

Table 1

Observed Parameters of the Sources within IRAS 14482-5857 at 1332, 1844, and $2868 \mathrm{MHz}$ as Marked in Figure 2

\begin{tabular}{|c|c|c|c|c|c|c|}
\hline Source & $\begin{array}{c}\text { Frequency } \\
(\mathrm{MHz}) \\
(2)\end{array}$ & $\begin{array}{c}\alpha \\
(\mathbf{J} 2000.0) \\
(3)\end{array}$ & $\begin{array}{c}\delta \\
(\mathrm{J} 2000.0) \\
(4)\end{array}$ & $\begin{array}{c}\text { Angular Size/P.A. } \\
(", \circ) \\
(5)\end{array}$ & $\begin{array}{c}I_{\nu} \\
(\mathrm{mJy} / \mathrm{beam}) \\
(6)\end{array}$ & $\begin{array}{c}S_{\nu} \\
(\mathrm{mJy}) \\
(7)\end{array}$ \\
\hline \multirow[t]{3}{*}{$\overline{\mathrm{A}}$} & 1332 & $14: 52: 04.26$ & $-59: 10: 03.0$ & $20.4 \times 18.5,-5.1$ & $51.9 \pm 0.3$ & $272 \pm 13$ \\
\hline & 1844 & 14:52:04.34 & $-59: 10: 01.9$ & $15.6 \times 13.1,-15.8$ & $45.2 \pm 0.2$ & $200 \pm 10$ \\
\hline & 2868 & $14: 52: 04.35$ & $-59: 10: 01.5$ & $11.9 \times 10.0,-16.0$ & $23.0 \pm 1.0$ & $155 \pm 8$ \\
\hline \multirow[t]{3}{*}{$\mathrm{B}$} & 1332 & $14: 52: 11.06$ & $-59: 09: 56.8$ & $106.1 \times 48.9,-71.1$ & $8.4 \pm 0.4$ & $606 \pm 30$ \\
\hline & 1844 & $14: 52: 11.51$ & $-59: 09: 56.8$ & $90.6 \times 44.1,-69.8$ & $4.8 \pm 0.2$ & $418 \pm 21$ \\
\hline & 2868 & $14: 52: 12.25$ & $-59: 10: 02.8$ & $07.9 \times 07.3,-46.7$ & $7.7 \pm 0.4$ & $25 \pm 1$ \\
\hline \multirow[t]{2}{*}{$\mathrm{C}$} & 1332 & $14: 52: 13.20$ & $-59: 09: 46.4$ & $43.1 \times 22.6,1.9$ & $6.1 \pm 0.3$ & $82 \pm 4$ \\
\hline & 1844 & $14: 52: 00.37$ & $-59: 09: 47.7$ & $20.1 \times 10.0,+29.0$ & $3.6 \pm 0.2$ & $16 \pm 1$ \\
\hline
\end{tabular}

Note. Units of R.A. are hours, minutes, and seconds and units of decl. are degrees, minutes, and seconds.

scales that these observations trace are not larger than the source, and hence we would only be subtracting source flux density. A more careful analysis would take this into account at shown here) does not reveal emission that follows the same morphology as Figure 5. This suggests that the material located near $0 \mathrm{~km} \mathrm{~s}^{-1}$ is associated with IRAS 14482-5857. On the other 


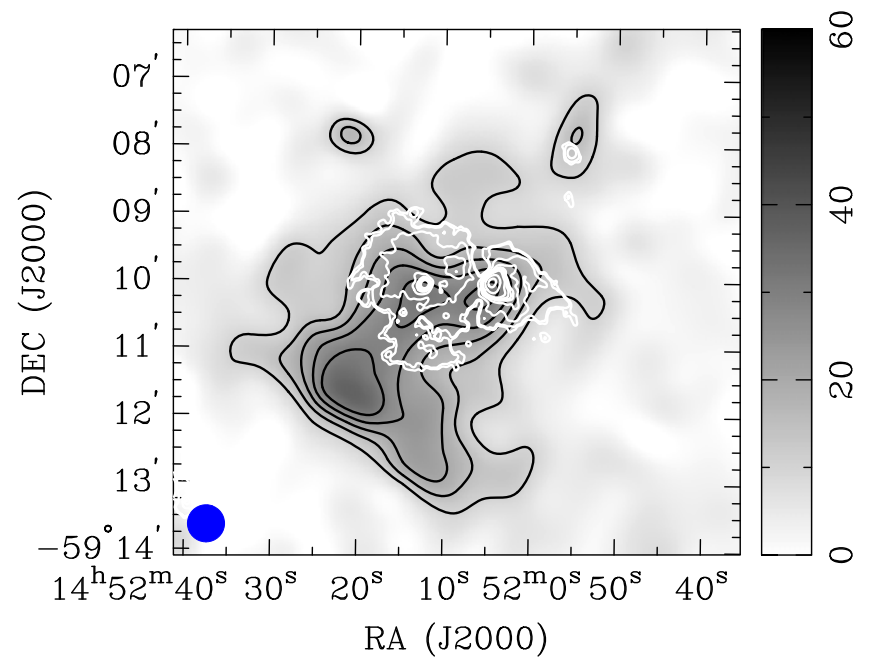

Figure 5. Integrated-intensity image of the region surrounding IRAS 144825857 obtained using the velocity-cube for the ${ }^{12} \mathrm{CO}$-line and integrated over the velocity range of -10 to $+10 \mathrm{~km} \mathrm{~s}^{-1}$, overlaid with the same contours at 10 , $15,20,25,30$, and $35 \mathrm{~K} \mathrm{~km} \mathrm{~s}^{-1}$ (the peak emission is at $35 \mathrm{~K} \mathrm{~km} \mathrm{~s}^{-1}$ ). Overlaid on the integrated-intensity image are (white) contours of our $1844 \mathrm{MHz}$ ATCA data, using the same contours as in Figure 2. The intensity range (for a linear scaling) runs from 0 to $60 \mathrm{~K} \mathrm{~km} \mathrm{~s}^{-1}$, so as to illustrate the peaks of emission coinciding with the radio continuum peaks. The $35^{\prime \prime}$ beam of the ${ }^{12} \mathrm{CO}$ emission is shown in lower left-hand corner.

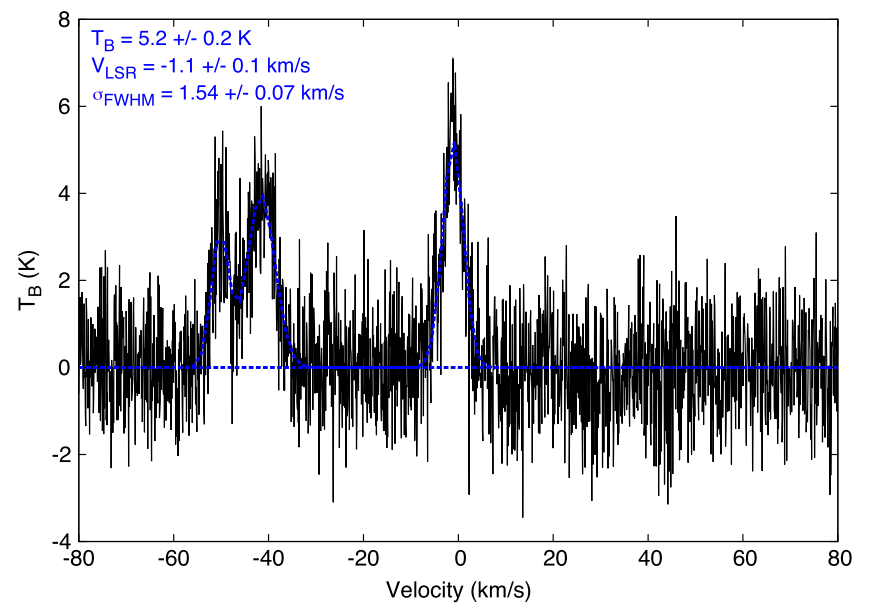

Figure 6. Brightness temperature vs. velocity plot for the ${ }^{12} \mathrm{CO}$ emission toward the peak of the emission found toward IRAS 14482-5857 as shown in Figure 5. The derived parameters for the fit to the data (as shown by the bluedashed line) for peaked emission at $-1.12 \pm 0.1 \mathrm{~km} \mathrm{~s}^{-1}$ are shown in the top left-hand corner of the plot.

hand, Figure 5 shows the positional coincidence of the peak of emission of both the ${ }^{12} \mathrm{CO}$ and radio continuum (peaks $\mathrm{A}$ and $\mathrm{B}$ of Figure 2). This figure also illustrates the extension of the molecular emission to the southeast of the radio source and its termination in a linear-like feature running roughly northeast to southwest and represented by the tight grouping of the contours found there.

Figure 6 shows the line profile for the ${ }^{12} \mathrm{CO}$ emission toward the peak of the radio emission at $1844 \mathrm{MHz}$, given as source $\mathrm{A}$ in Figure 2 (right). We have fit (using an iterative $\chi^{2}$ fitting procedure) the resulting spectrum for this position and find that a two-plus-one component fit represents the data best. We fit a two-component Gaussian to the $\sim-42 \mathrm{~km} \mathrm{~s}^{-1}$ line emission, one at $V_{\mathrm{LSR}}=-41.6 \pm 0.2 \mathrm{~km} \mathrm{~s}^{-1}$ with a brightness temperature of $T_{\mathrm{mb}}=4.0 \pm 0.1 \mathrm{~K}$ and an FWHM of

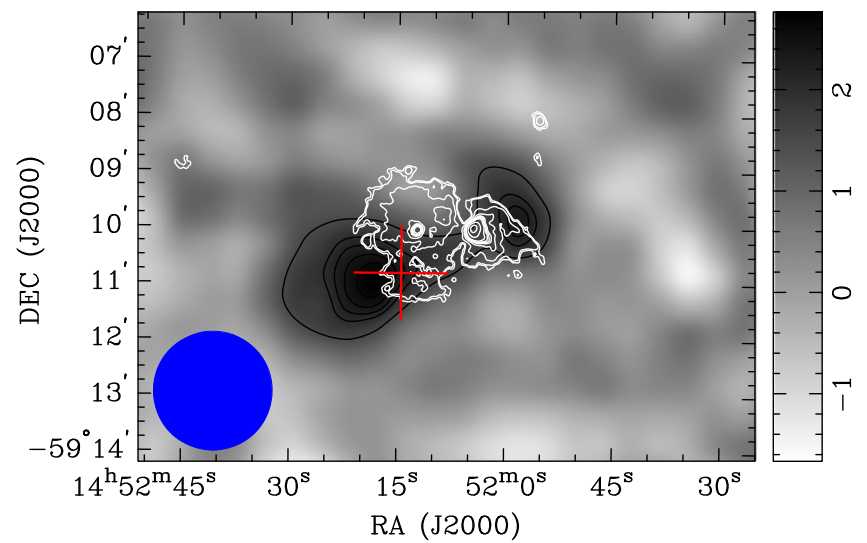

Figure 7. Velocity-integrated image of $22.2 \mathrm{GHz} \mathrm{H}_{2} \mathrm{O}$ maser emission surrounding IRAS 14482-5857. The emission seen here has been integrated between -10 and $+10 \mathrm{~km} \mathrm{~s}^{-1}$ and is overlaid with the same (black) contours at $1.5,2,2.2,2.4$, and $2.6 \mathrm{~K}$. The (red) cross represents the maser emission reported by the HOPS collaboration (Walsh et al. 2011). The (white) contours are $1844 \mathrm{MHz}$ radio continuum emission at $0.8,1,2,4,8$, and $16 \mathrm{mJy} / \mathrm{beam}$. The $2^{\prime}$ Mopra beam for the HOPS data is shown in the lower left-hand corner.

$\sigma_{\text {FWHM }}=2.1 \pm 0.1 \mathrm{~km} \mathrm{~s}^{-1}$. The second peak is fit using a brightness temperature of $T_{\mathrm{mb}} \sim 3.1 \pm 0.2 \mathrm{~K}$ at a velocity of $V_{\mathrm{LSR}}=-50.2 \pm 0.2 \mathrm{~km} \mathrm{~s}^{-1}$ at an FWHM of $\sigma_{\text {FWHM }}=1.3 \pm 0.1 \mathrm{~km} \mathrm{~s}^{-1}$. The peak of emission at $\sim 0 \mathrm{~km} \mathrm{~s}^{-1}$ is fit using a brightness temperature of $T_{\mathrm{mb}}=5.2 \pm 0.2 \mathrm{~K}$ at a velocity of $V_{\mathrm{LSR}}=-1.1 \pm 0.1 \mathrm{~km} \mathrm{~s}^{-1}$ and an FWHM width of $\sigma_{\mathrm{FWHM}}=1.5 \pm 0.1 \mathrm{~km} \mathrm{~s}^{-1}$. This fitting shows that, in addition to the peak-velocity analysis described above, the emission component at $V_{\mathrm{LSR}}=-1.1 \pm 0.1 \mathrm{~km} \mathrm{~s}^{-1}$ is stronger than the $V_{\mathrm{LSR}}=-41.6,-50.2 \mathrm{~km} \mathrm{~s}^{-1} \quad$ components. Using the ${ }^{13} \mathrm{CO}$ data cube, we have similarly fit the emission from the peak of the radio continuum. Although the weaker nature of the lines here means that we could not reliably fit any emission around the $-42 \mathrm{~km} \mathrm{~s}^{-1}$ line seen in the ${ }^{12} \mathrm{CO}$ cube, we could fit a component to the emission around $-1.1 \mathrm{~km} \mathrm{~s}^{-1}$. Doing this results in parameters of $T_{\mathrm{mb}} \sim 1.4 \pm 0.1 \mathrm{~K}$ at a velocity centroid of $V_{\mathrm{LSR}}=-0.9 \pm 0.1 \mathrm{~km} \mathrm{~s}^{-1}$ and an FWHM width of $\sigma_{\text {FWHM }}=1.0 \pm 0.1 \mathrm{~km} \mathrm{~s}^{-1}$. Using the velocity at peak intensity for each of the components in the ${ }^{12} \mathrm{CO}$ data and assuming that IRAS 14482-5857 is indeed associated with these clouds, we derive near (far) distance estimates of $\sim 3.5(9.0) \mathrm{kpc}$ and $2.85(9.75) \mathrm{kpc}$ for the -50.2 and $-41.6 \mathrm{~km} \mathrm{~s}^{-1}$ peaks, respectively, and $\sim 12.7 \mathrm{kpc}$ for the $-1.1 \mathrm{~km} \mathrm{~s}^{-1}$ peak.

\subsection{3. $\mathrm{H}_{2} \mathrm{O}$ Maser and Ammonia Line Emission}

From the HOPS data and catalog (Walsh et al. 2011), we find that a water maser source with a peak flux density of $5.1 \mathrm{Jy}$ at $-3.3 \mathrm{~km} \mathrm{~s}^{-1}$ with a line width of $1.0 \mathrm{~km} \mathrm{~s}^{-1}$ is cataloged at a position of (R.A., decl.; J2000.0) = $14^{\mathrm{h}} 52^{\mathrm{m}} 14^{\mathrm{s}} .5,-59^{\circ} 10^{\mathrm{m}} 49^{\mathrm{s}}$. 0 . From the given conversion factor of 12.5 at $22.2 \mathrm{GHz}$, this flux corresponds to a main beam brightness temperature of $0.4 \mathrm{~K}$.

Figure 7 shows the velocity-integrated emission (integrated between -10 and $+10 \mathrm{~km} \mathrm{~s}^{-1}$ ) toward IRAS 14482-5857. In addition to the position of the maser emission reported in Walsh et al. (2011), which is described by the (red) cross, it shows two regions of possible maser emission. The first peak (at $\sim 2.7 \mathrm{~K} \mathrm{~km} \mathrm{~s}^{-1}$ ) is located at $\alpha=14^{\mathrm{h}} 52^{\mathrm{m}} 17^{\mathrm{s}} .8$, $\delta=-59^{\circ} 10^{\prime} 50$ !' $2 \quad(\mathbf{J} 2000.0)$ and is the maser emission 
reported above. The second peak (at $\sim 2.3 \mathrm{~K} \mathrm{~km} \mathrm{~s}^{-1}$ ) is located at $\alpha=14^{\mathrm{h}} 51^{\mathrm{m}} 58.3, \delta=-59^{\circ} 09^{\prime} 50^{\prime \prime} \cdot 2 \quad(\mathrm{~J} 2000.0)$, which is located at a $\sim 2$ !. 7 angular distance from the reported maser spot, which is a separation of about 1.2 beams. Thus, we take only the listed brightness temperature, velocity, and velocity width listed in Walsh et al. (2011), suggesting that observations with a higher angular resolution instrument such as the ATCA are required to reveal the nature of the $\mathrm{H}_{2} \mathrm{O}$ maser emission throughout IRAS 14482-5857.

Using a velocity centroid of $v=-3.3 \mathrm{~km} \mathrm{~s}^{-1}$ and assuming that this source is associated with the radio emission, we arrive at a distance to IRAS $14482-5857$ of $12.5 \mathrm{kpc}$. This is similar to the distance derived above from the ${ }^{12} \mathrm{CO}$ emission, and hereafter, we adopt a distance to IRAS $14482-5857$ of $12.5 \mathrm{kpc}$. This implies that the source extent results in an intrinsic source size of $\sim 11 \times 14.5 \mathrm{pc}$.

Utilizing the publicly available data cubes from the HOPS website, ${ }^{5}$ we have searched for emission from the inversion transitions of the $\mathrm{NH}_{3}(1,1),(2,2)$, and $(3,3)$ lines. We found no emission coincident with the IRAS 14482-5857 source at either velocity down to the limiting $\sigma_{T_{\mathrm{mb}}}=0.20 \pm 0.06 \mathrm{~K}$. For the near distance, HOPS would observe a $400 M_{\odot}$. cloud here with a $5 \sigma$ level (whereas this increases to $\sim 10^{4} M_{\odot}$ at the far distance). Interestingly, for the $(1,1)$ transition, this equates to a $3.2 \mathrm{kpc}$ distance limit for detecting a $20 \mathrm{~K}, 400 M_{\odot}$ cloud at the $5 \sigma$ level with more massive clouds being observable farther away with a $3 \times 10^{4} M_{\odot}$ cloud being observed anywhere in the Galaxy. We note that even though $\mathrm{H}_{2} \mathrm{O}$ maser emission has been known to appear at velocities far from the systemic velocity of the source that created it (Breen et al. 2010a), the strong ${ }^{12} \mathrm{CO}$ lines fitted in the previous section suggest that this is not the case here.

\subsubsection{Other Masing Emission Surveys}

Maser searches, particularly the $6.7 \mathrm{GHz}$ methanol maser line first detected by Menten (1991) over 20 years ago, are excellent discriminators of massive star-forming regions. Indeed, it has recently been shown that this masing transition is exclusively associated with massive star formation (Breen et al. 2013). However, surveys searching for $6.7 \mathrm{GHz}$ methanol masers have not detected any significant maser emission from the region down to a limiting flux density of $0.3 \mathrm{Jy}$, for a beam size of 3!3 (Walsh et al. 1997). We have searched the literature for other observations of this region using other maser tracers, namely class I methanol masers at 33 and $44 \mathrm{GHz}$ (Voronkov et al. 2014). No survey other than HOPS has reported maser emission from IRAS 14482-5857 (i.e., the methanol maser surveys, e.g., Caswell 1998; Green et al. 2012).

\subsection{Infrared Emission}

Figure 8 presents a three-color image of IRAS 14482-5857 at 3.6 (blue; GLIMPSE/IRAC), 8 (green; GLIMPSE/IRAC), and $24 \mu \mathrm{m}$ (red; MSX/MIPS).

This image shows a complex source morphology. The medium surrounding IRAS 14482-5857 is dominated by $8 \mu \mathrm{m}$ emission, which is sensitive to $\mathrm{PAH}$ emission, and a strong tracer of massive star formation (Tielens 2008). This PAH emission is wispy and filamentary in nature, extending some $\sim 1-2^{\prime}$ further than and appearing to confine the radio

\footnotetext{
http://awalsh.ivec.org/hops/public/index.php
}

emission. The extension of the diffuse radio continuum emission toward the southwest and southeast of the source follows minima of the PAH emission. Hot dust emission, traced by the $24 \mu \mathrm{m}$ emission, is most obviously seen within the diffuse radio filled bubble morphology of a classical HII region of this source, although it is mixed with significant amounts of PAH emission, giving large parts of the bubbles the yellow color seen in the image. The southwest bubble extends farther out and matches low surface brightness emission traced by hot dust emission.

Figure 9 shows a closeup of the radio bubble structures of IRAS 14482-5857. The levels in this figure have been adjusted to bring out the detail in the emission in the bubble, which was saturated in Figure 8, so as to illustrate the low surface brightness and filamentary nature of the PAH emission surrounding the object. Figure 9 shows that the southeast bubble is dominated by dim emission from hot dust, which is surrounded by PAH emission, particularly toward the center of the source, where the filled bubbles are observed to pinch together in the radio continuum. This behavior is also seen in the western bubble, although it is smaller and the dust emission is brighter and dominated by a strong point source at $24 \mu \mathrm{m}$. The central regions of IRAS 14482-5857 again show dust- or $\mathrm{PAH}$-dominated emission, which is aligned in a direction perpendicular to the extension of the diffuse radio bubble.

Figure 10 shows a closeup of the central regions of IRAS 14482-5857. It reveals that at the point where the radio bubbles pinch and meet is a blue, compact source dominated by $3.6 \mu \mathrm{m}$ emission, suggesting that this is a young stellar object (YSO). This object appears to be surrounded by a cocoon of hot dust emission with at least one linear feature, reminiscent of an outflow that is yellow in color, suggesting that it results primarily from PAH and hot dust emission. It is interesting, and maybe significant, that this outflow-like structure is pointing toward the strongest peak of radio emission in the western bubble. A commensurate feature is not observed, however, toward the eastern bubble, which exhibits very low surface brightness PAH emission, again in the direction perpendicular to the bubble structures observed in our radio continuum data.

Figure 11 shows the region at $4.5 \mu \mathrm{m}$, thought to be due to shocked molecular hydrogen and $\mathrm{CO}$ band heads, which possibly indicates the presence of an outflow (Hindson et al. 2012). It is instructive, then, that the $4.5 \mu \mathrm{m}$ emission from this region is bright and concentrated near the central compact object (seen as the bright blue emission in Figure 10) and the brightest of the radio continuum emission regions. This perhaps demonstrates that this object possesses an outflow (Cyganowski et al. 2008).

Table 2 summarizes the flux densities in the far-infrared (FIR) waveband derived from the integration within the lowest contour of the radio emission at $1844 \mathrm{MHz}$. We find flux densities of 15, 10, 27, and $43 \mathrm{Jy}$ at the A, C, E, and D bands of $M S X$

\section{DISCUSSION}

\subsection{Spectral Energy Distribution (SED)}

Figure 12 shows the spectral energy distribution of IRAS $14482-5857$ from $843 \mathrm{MHz}$ up to $3.6 \mu \mathrm{m}$. The SED shows that the emission at FIR frequencies longward of $\sim 8 \mu \mathrm{m}$ is due to thermal dust emission, while the radio continuum emission is well fitted with an optically thin thermal bremsstrahlung model. 


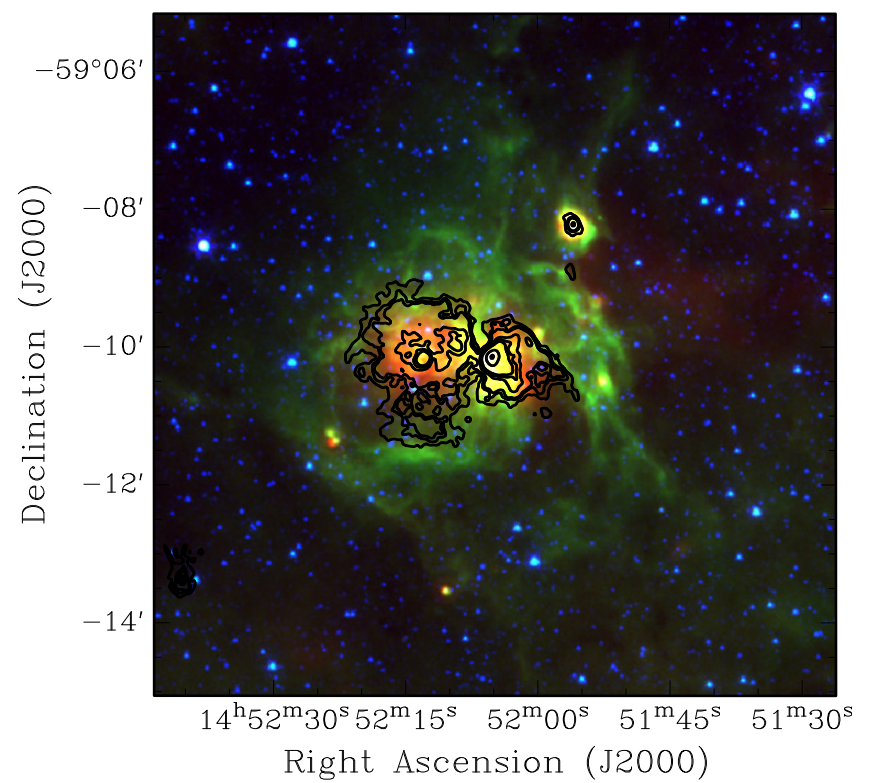

Figure 8. Three-color image of the region surrounding IRAS 14482-5857 obtained using $3.6 \mu \mathrm{m}$ (blue), $8 \mu \mathrm{m}$ (green) data from the GLIMPSE survey, as well as $24 \mu \mathrm{m}$ (red) data from MIPS. The $24 \mu \mathrm{m}$ emission runs from 6 to $162 \mathrm{MJy} \mathrm{sr}^{-1}$, while the 3.6 and $8 \mu \mathrm{m}$ emission runs from 0.3 to 24.5 and 27 to $127 \mathrm{MJy} \mathrm{sr}^{-1}$, respectively, under a linear transfer function. The contours, now black and at levels starting at $0.8 \mathrm{mJy} /$ beam increase as $0.8^{*} 2^{n}$ for $n \in[1,8]$, in order to maximize clarity in the three-color image.

The FIR and dust emission were fitted with a two-component "graybody" model, which is a modified blackbody curve where the opacity is assumed to vary with frequency as $\tau_{\nu}=\left(\nu / \nu_{0}\right)^{\beta}$, where $\nu_{0}$ is the frequency at which the optical depth of that component is unity. The flux density is then modeled according to $S_{\nu}=B_{\nu}\left(T_{\mathrm{d}}\right)\left[1-\exp \left(-\tau_{\nu}\right)\right] \Omega_{\mathrm{S}}$, where $B_{\nu}\left(T_{\mathrm{d}}\right)$ is the Planck function at the dust temperature, $T_{\mathrm{d}}$, and $\Omega_{\mathrm{S}}$ is the solid angle subtended by the dust-emitting part of the source (i.e., the region interior to the $\mathrm{PAH}$ emission in Figure 8 and commensurate with the lowest radio continuum contour). In the fitting, we have used an opacity index, $\beta$, of 2.0 following Garay et al. (2002) for sources with limited spectral points and consistent for sources showing signs of high-mass star formation (Ossenkopf \& Henning 1994). We find a cold dust component at $19 \mathrm{~K}$ for an angular size of $3^{\prime} \times 4^{\prime}$, assuming a Gaussian flux distribution within the source and an optical depth of unity at $27 \mu \mathrm{m}$. The hot dust component is well-fitted with a temperature of $110 \mathrm{~K}$.

The radio continuum emission below $\sim 100 \mathrm{GHz}$ is well fit with an optically thin free-free emission and to fit the emission, we used a technique of "bootstrapping" the thermal flux density (Crocker et al. 2010 supplementary information) to that given by the $4500 \mathrm{MHz}$ PMN $1452-5910$ datum, F(4500 MHz), assuming that it is completely thermal, so that the optical depth at $4500 \mathrm{MHz}, \tau(4500 \mathrm{MHz})$ is given by:

$$
\tau(4500 \mathrm{MHz})=\frac{F(4500 \mathrm{MHz})}{\Omega_{\mathrm{S}} B(4500 \mathrm{MHz}, T)},
$$

where $\Omega_{\mathrm{S}}$ is the solid angle of the source and $B(4500 \mathrm{MHz}, T)$ is the Planck function at temperature, $T$. We then obtain the

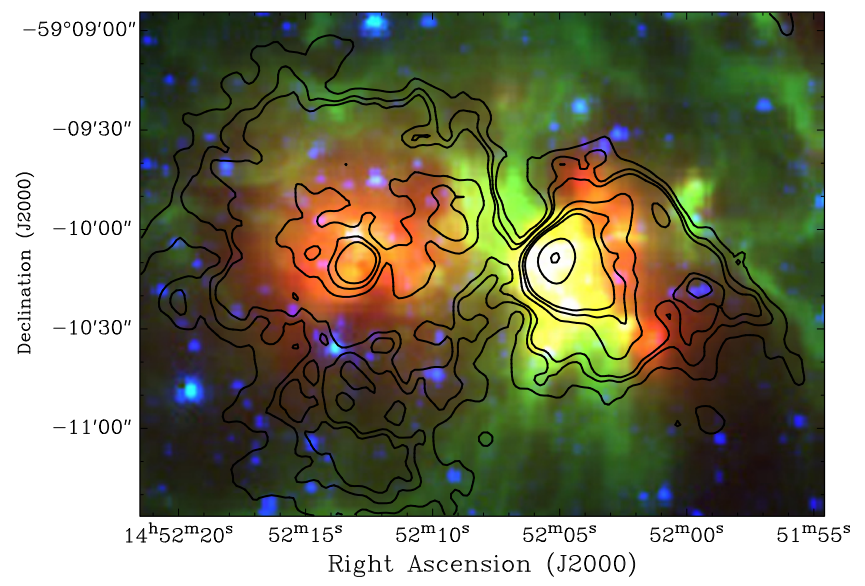

Figure 9. Closeup of the diffuse radio emission of IRAS 14482-5857. The colors are the same as in Figure 8, with the red and green levels adjusted to bring out specific features observed within the bubble. Specifically the $24 \mu \mathrm{m}$ levels are now from 6 to $237.5 \mathrm{MJy} \mathrm{sr}^{-1}$, while the $8 \mu \mathrm{m}$ emission is from 28 to $154 \mathrm{MJy} \mathrm{sr}^{-1}$. The radio contours are the same $1844 \mathrm{MHz}$ ATCA contours as in Figure 8 .

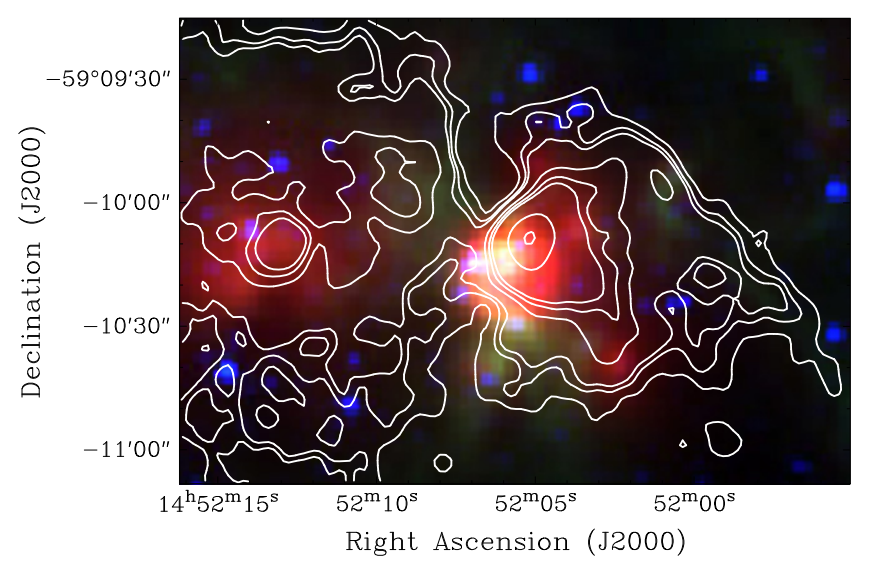

Figure 10. Closeup of the central region of IRAS 14482-5857. The colors are the same as in Figure 8, with the levels adjusted to bring out specific features observed within central parts of the source. Specifically the $24 \mu \mathrm{m}$ levels are now from 30 to $540 \mathrm{MJy} \mathrm{sr}^{-1}$, while the 8 and $3.6 \mu \mathrm{m}$ emission runs from 25 to $550 \mathrm{MJy} \mathrm{sr}^{-1}$ and 20 to $40 \mathrm{MJy} \mathrm{sr}^{-1}$, respectively. The (white) radio contours are the same $1844 \mathrm{MHz}$ ATCA contours as in Figure 8.

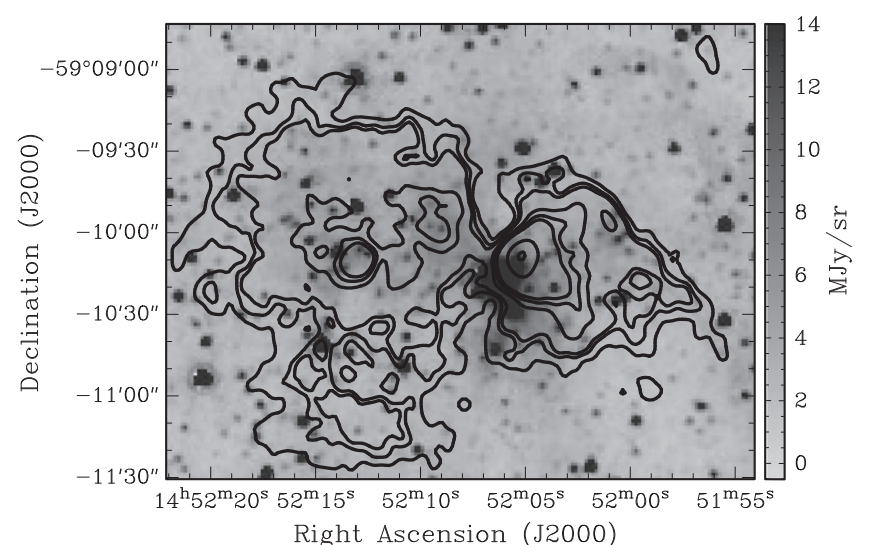

Figure 11. GLIMPSE $4.5 \mu \mathrm{m}$ image of IRAS 14482-5857, overlaid with same (white) ATCA contours as in Figure 8. 


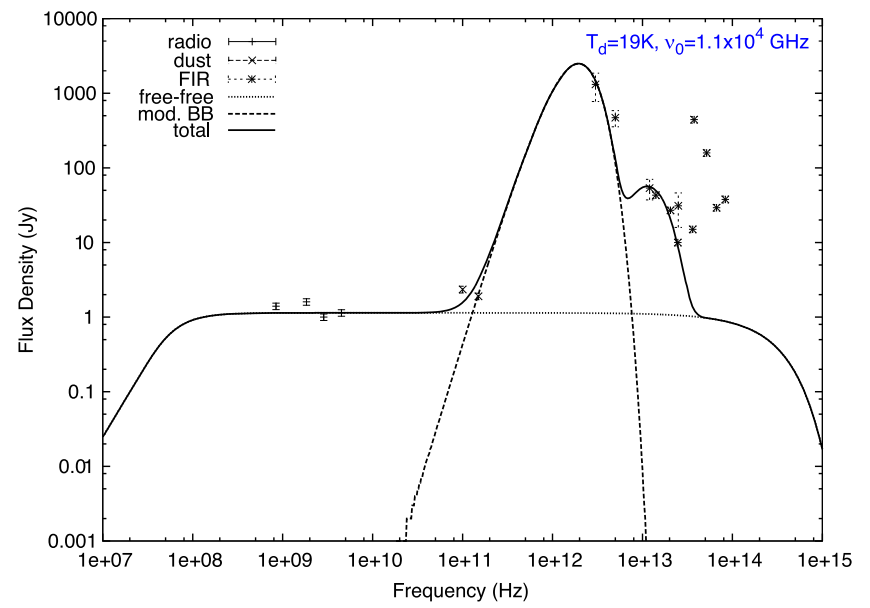

Figure 12. Total spectral energy distribution (SED; solid black line) of IRAS 14482-5857 from the radio continuum through to the IR. Plus signs mark the radio $(843-4500 \mathrm{MHz})$; crosses note the dust emission at 100 and $150 \mathrm{GHz}$ from Culverhouse (2011); and stars mark the IRAS, MSX, and GLIMPSE fluxes as described in Table 2. The dotted curve is optically thin thermal bremsstrahlung emission modeled by "bootstrapping" the flux to the $4500 \mathrm{MHz}$ PMN datum (see the text for more details). The dashed curve is a fit to the spectrum using two modified blackbody functions of the form $B_{\nu}\left(T_{\mathrm{d}}\right) 1-\exp \left[-\left(\nu / \nu_{0}\right)^{\beta}\right]$ with different temperatures. The fit parameters for the colder component are shown in the top right-hand corner.

Table 2

Observed Parameters of the Sources within IRAS 14482-5857 in the FIR

\begin{tabular}{lcc}
\hline \hline $\begin{array}{l}\text { Frequency } \\
(\mu \mathrm{m})\end{array}$ & $\begin{array}{r}\text { Average Flux Density } \\
\left(\mathrm{Jy} \mathrm{sr}^{-1}\right)\end{array}$ & $\begin{array}{c}\text { Integrated Flux Density } \\
(\mathrm{Jy})\end{array}$ \\
$(1)$ & $(2)$ & $(3)$ \\
\hline $3.6^{\mathrm{a}}$ & 6.3 & 38 \\
$4.5^{\mathrm{a}}$ & 4.9 & 29 \\
$5.8^{\mathrm{a}}$ & 26.4 & 158 \\
$8.0^{\mathrm{a}}$ & 73.7 & 443 \\
$8.28^{\mathrm{b}}$ & 14.8 & 15 \\
$12.0^{\mathrm{c}}$ & 30.7 & 31 \\
$12.13^{\mathrm{b}}$ & 9.9 & 10 \\
$14.65^{\mathrm{b}}$ & 26.7 & 27 \\
$21.34^{\mathrm{b}}$ & 42.7 & 43 \\
$25^{\mathrm{c}}$ & 53.7 & 54 \\
$60^{\mathrm{c}}$ & 192 & 470 \\
$100^{\mathrm{c}}$ & 1302 & 1315 \\
\hline
\end{tabular}

a IRAC/GLIMPSE data.

b $M S X$.

c IRAS.

optical depth at frequency $\nu$ from:

$$
\tau_{\nu}=\tau(4500 \mathrm{MHz})\left(\frac{\nu}{10 \mathrm{GHz}}\right)^{-2.1}
$$

Hence the flux density, $S_{\nu}$ at any frequency is:

$$
S_{\nu}=\Omega_{\mathrm{S}} B(4500 \mathrm{MHz}, T)\left[1-e^{-\tau_{\nu}}\right] .
$$

Using a nominal electron temperature of $10,000 \mathrm{~K}$, we fit the thermal emission. The total luminosity radiated by the source as a whole, found by integrating the emission shown in Figure 12 from $10 \mathrm{MHz}$ up to $1 \mathrm{THz}$, is $\sim 3 \times 10^{5} L_{\odot}$.

\subsection{Derived Parameters}

\subsubsection{Mass Estimate from Dust Emission}

Following Garay et al. (2002), the fact that the thermal dust emission at $150 \mathrm{GHz}$ is optically thin implies that the mass of the cloud, $M_{\mathrm{g}}$ can be estimated:

$$
M_{\mathrm{g}}=\frac{S_{\nu} D^{2}}{R_{\mathrm{dg}} \kappa_{\nu} B_{\nu}\left(T_{\mathrm{d}}\right)},
$$

where $\kappa_{\nu}$ is the mass absorption coefficient of the dust, $R_{\mathrm{dg}}$ is the dust-to-gas ratio (assuming 10\% helium), and $B_{\nu}\left(T_{\mathrm{d}}\right)$ is the Planck function at the dust temperature, $T_{\mathrm{d}}$. Using the same values for the above equation as found in (Garay et al. 2002), we arrive at a mass estimate for IRAS 14482-5857, parameterized as a function of the assumed distance of $12.7 \mathrm{kpc}$ (as suggested by the ${ }^{12} \mathrm{CO}$ analysis in Section 3.2.2) of:

$$
M_{\mathrm{g}} \sim 1.3 \times 10^{4}\left(\frac{D}{12.7 \mathrm{kpc}}\right)^{2} M_{\odot} .
$$

It must be explicitly noted, however, that even assuming a distance of $12.7 \mathrm{kpc}$, the above determination is dependant on $R_{\mathrm{dg}} \kappa_{\nu}$, which is highly uncertain (Garay et al. 2002). Hence we have also obtained mass estimates for IRAS 14482-5857 from the ${ }^{12} \mathrm{CO}$ data.

\subsection{2. ${ }^{12} \mathrm{CO}$ and ${ }^{13} \mathrm{CO}$ Optical Depth Analysis and Mass Estimates}

From the fitting of the ${ }^{12} \mathrm{CO}$ and ${ }^{13} \mathrm{CO}$ line presented in Section 3.2.2, we obtain an estimate of the optical depth of the ${ }^{13} \mathrm{CO}$ line from:

$$
\tau\left({ }^{13} \mathrm{CO}\right)=\frac{T_{\mathrm{A}}\left({ }^{13} \mathrm{CO}\right)}{T_{\mathrm{A}}\left({ }^{12} \mathrm{CO}\right)} \sim 0.3,
$$

where $T_{\mathrm{A}}\left({ }^{13} \mathrm{CO}\right)$ are the fitted brightness temperatures obtained from fitting the line profiles presented in Figure 6 and assumed to be representative of the cloud as a whole. Assuming a ratio of carbon 12 to its isotope of $X_{12 / 13}=54$ (Burton et al. 2013), we then obtain an optical depth for the main line of $\tau\left({ }^{12} \mathrm{CO}\right)=\tau\left({ }^{13} \mathrm{CO}\right) X_{12 / 13} \sim 14$. This shows that the ${ }^{12} \mathrm{CO}$ line is optically thick, but that the ${ }^{13} \mathrm{CO}$ is optically thin for this cloud. Using the empirical relation describing the CO-to- $\mathrm{H}_{2}$ conversion factor known as the $X$-factor from Bolatto et al. (2013) of $X^{{ }^{12} \mathrm{CO}}=2 \times 10^{20} \mathrm{~cm}^{-2}(\mathrm{~K} \mathrm{~km} \mathrm{~s})^{-1}$, we find a mass for the cloud of $\sim 2 \times 10^{4} M_{\odot}$, similar to that derived from the dust flux densities in the previous section. We note that a more detailed analysis, such as that laid out in Burton et al. (2013) (and references therein), or virial mass estimates using the line parameters described in Section 3.2.2, also arrive at a similar mass. This mass also suggests the reason why the HOP survey did not observe $\mathrm{NH}_{3}$ emission toward this source.

\subsection{Radio and FIR-derived Parameters}

Sections 3.2.2 and 3.2.3 strongly suggest that the emission from this region comes from a distance of $\sim 12.7 \mathrm{kpc}$. This implies spatial dimensions for IRAS 14482-5857 $r \sim 11 \times 14.7 \mathrm{pc}$ for an angular source extent of $3^{\prime} \times 4^{\prime}$. Figures 2 and 3 and Table 1 also show that there are three bright peaks of radio emission embedded within IRAS 144825857. Here we derive simple parameters for these regions 
Table 3

Derived Parameters of the UCHII Regions

\begin{tabular}{lccccl}
\hline \hline Region & $\begin{array}{c}\text { Size } \\
-\end{array}$ & $\begin{array}{c}\text { EM } \\
\left.(\mathrm{pc} \mathrm{cm})^{-6}\right)\end{array}$ & $\begin{array}{c}n_{\mathrm{e}} \\
\left(\mathrm{cm}^{-3}\right)\end{array}$ & $\begin{array}{c}N_{\text {Ly }} \\
\left(\mathrm{s}^{-1}\right)\end{array}$ & $\begin{array}{l}\text { Spectral } \\
\text { Type } \\
(6)\end{array}$ \\
\hline All & $(2)$ & $(3)$ & $(4)$ & $(5)$ & $(6)$ \\
A & $0.95 \times 0.80$ & $5 \times 10^{6}$ & $2.3 \times 10^{3}$ & $3 \times 10^{46}$ & B0 \\
B & $0.48 \times 0.43$ & $3.5 \times 10^{7}$ & $8.8 \times 10^{3}$ & $5 \times 10^{48}$ & O8 \\
C & $1.2 \times 0.61$ & $4 \times 10^{5}$ & $7 \times 10^{2}$ & $2 \times 10^{47}$ & B0 \\
\hline
\end{tabular}

assuming (as the spectral index maps in Figure 4 and integrated flux densities suggest) that these regions are HII regions. We also derive the same parameters for the region as a whole.

From the source spectrum derived in the previous section and shown in Figure 12, we find that IRAS 14482-5857 has an emission measure of $\sim 1.4 \times 10^{5} \mathrm{pc} \mathrm{cm}^{-6}$, an electron number density of $\sim 96 \mathrm{~cm}^{-3}$, and an ionizing photon production rate of $\sim 7 \times 10^{47} \mathrm{~s}^{-1}$. Assuming that the region as a whole is excited by a single zero-age main sequence star, it would be of spectral type B0.5. Table 3 shows the parameters derived for each of the compact sources within IRAS 14482-5857. This shows that IRAS 14482-5857 consists of three UCHII regions (the bright peak sources A, B, and C) embedded within an Hir region. It also shows that if the components that can be classed as UCHII regions are excited by individual zero-age main sequence stars, that the rates of UV photons required to ionize them imply spectral types of said stars of at least B0/1, except for source B, which is strong and compact, and at this assumed distance implies a star of spectral type O8.

\subsection{The Nature of IRAS 14482-5857}

The nature of IRAS 14482-5857, based on the evidence elucidated above, is strongly suggestive of massive star formation. Walsh et al. (1997) suggest that this source is a UCHII region based on the IRAS colors and the WoodChurchwell relation. However, several studies of $6.7 \mathrm{GHz}$ methanol maser emission toward this source failed to find any emission down to a limiting flux of $300 \mathrm{mJy} / \mathrm{beam}$ (compare Section 3.2.4). We suggest that this resulted in this source being overlooked as a site of star formation. Evidence of IRAS $14482-5857$ being a site of massive star formation is indicated by the following.

1. ${ }^{12} \mathrm{CO}$ and ${ }^{13} \mathrm{CO}$ observations showing strong emission toward the peaks of the radio continuum, which are dominated by emission at a velocity centroid of $V_{\mathrm{LSR}}=-1.1 \mathrm{~km} \mathrm{~s}^{-1}$, with the ${ }^{13} \mathrm{CO}$ line not being able to be fitted for the velocity centroids at $V_{\mathrm{LSR}}=-41.6$ and $-50.2 \mathrm{~km} \mathrm{~s}^{-1}$ at all (though we note that this is probably due to the noise in the ${ }^{13} \mathrm{CO}$ cubes. The derived (hydrogen) column density and mass (viz. $\left.N_{\mathrm{H}_{2}} \sim 1.5 \times 10^{21} \mathrm{~cm}^{-2} \sim 2 \times 10^{4} M_{\odot}\right)$ ) are commensurate with massive star-forming regions.

2. The presence of $\mathrm{H}_{2} \mathrm{O}$ maser emission toward this source (see Section 3.2.3). The presence of the strong ${ }^{12} \mathrm{CO}$ emission at a velocity centroid of $V_{\mathrm{LSR}}=-1.1$ $\mathrm{km} \mathrm{s}^{-1}$ suggests that the velocity of the maser is not significantly displaced from the velocity of the starforming region itself.

3. A dust-derived cloud mass and temperature of $\sim 10^{5} M$. and $110 \mathrm{~K}$, respectively.
4. The implied size, emission measure, electron number density, and ionizing photon production rate derived from the strong, compact radio continuum sources are representative of UCHII regions ionized by $\mathrm{O} / \mathrm{B}$ stars.

5. The association of the brightest UCHII region with a bright $4.5 \mu \mathrm{m}$ source, which may be producing outflowlike structures observed at 8 and $24 \mu \mathrm{m}$.

We note that the image in Figure 11 is subject to projection effects that may mimic such an outflowlike structure, so that this feature should be investigated further, but is nevertheless interesting. The UCHII regions are surrounded by diffuse radio emission that itself can be considered an HII region. This suggests, along with the bi-lobal radio structure that is seen perpendicular to the strong 8 and $24 \mu \mathrm{m}$ emission, that this source is a site of massive star formation at an advanced stage. One possible objection to this hypothesis is that there are no class 1 methanol masers, which are known to be exclusively associated with high mass star formation (Breen et al. 2013). However, the well known star-forming region, Orion Source I, which is forming massive stars, does not contain methanol maser emission (Goddi et al. 2009; Matthews et al. 2010).

\section{CONCLUSIONS}

IRAS 14482-5857 has been the subject of few searches for southern sites of star formation; even though it possessed IRAS colors suggestive of star formation, as the lack of $6.7 \mathrm{GHz}$ methanol maser emission (down to a limiting flux density of $300 \mathrm{mJy} / \mathrm{beam}$ ) meant that this source was not investigated further. We have presented here new evidence that indeed IRAS $14482-5857$ is a site of massive star formation. Our results can be summarized as follows.

1. We have presented new, serendipitous ATCA observations of IRAS $14482-5857$ at $1-3 \mathrm{GHz}$ that are an order of magnitude higher in resolution $\left(\sim 10^{\prime \prime}\right)$ and sensitivity $(\sim 100 \mu \mathrm{Jy} / \mathrm{beam})$ than available archival data. These observations reveal that this source possesses a complex, bi-lobal morphology that is, spectrally, dominated by optically thin free-free emission in the broad, low surface brightness bubbles, strongly suggestive of an extended, classical HII region. Embedded within this extended envelope are three compact sources that are suggestive of UCHII regions.

2. Spurred by the complex radio morphology, we have performed a detailed literature search and assembled a data set that includes infrared data from 3.5 to $100 \mu \mathrm{m}$, radio continuum to millimeter radio data from $843 \mathrm{MHz}$ to $150 \mathrm{GHz}$, as well as maser and molecular line data from the HOPS and Mopra Southern Galactic Plane CO Surveys.

3. The molecular line data, principally the Mopra Southern Galactic Plane CO Survey data, reveal a complex morphological structure. Toward the brightest radio peak, the spectrum of the ${ }^{12} \mathrm{CO}$ emission exhibits three peaks: two near $V_{\mathrm{LSR}} \sim-42 \mathrm{~km} \mathrm{~s}^{-1}$ and one near $V_{\mathrm{LSR}} \sim 0$ $\mathrm{km} \mathrm{s}^{-1}$. The-albeit noisier- $-{ }^{13} \mathrm{CO}$ spectrum, however, exhibits only a single-peak structure centered near the $V_{\mathrm{LSR}} \sim 0 \mathrm{~km} \mathrm{~s}^{-1}$ component of the ${ }^{12} \mathrm{CO}$ spectrum.

4. Fitting the ${ }^{12} \mathrm{CO}$ and ${ }^{13} \mathrm{CO}$ spectra, we find that the (dimmer) peak at $\sim-42 \mathrm{~km} \mathrm{~s}^{-1}$ line emission can be fit by a two-component Gaussian with velocity centroids at $V_{\mathrm{LSR}}=-41.6 \pm 0.2 \mathrm{~km} \mathrm{~s}^{-1}$ and $V_{\mathrm{LSR}}=-50.2 \pm 0.2$ 
$\mathrm{km} \mathrm{s}^{-1}$. These peaks possess a brightness temperature of $T_{\mathrm{mb}}=4.0 \pm 0.1 \mathrm{~K}$ and a FWHM of $\sigma_{\mathrm{FWHM}}=1.3 \pm 0.1$ $\mathrm{km} \mathrm{s}^{-1}$ for the former, and $T_{\mathrm{mb}}=3.1 \pm 0.2 \mathrm{~K}$ and $\sigma_{\text {FWHM }}=1.5 \pm 0.1 \mathrm{~km} \mathrm{~s}^{-1}$ for the latter peak. We find a bright peak of ${ }^{12} \mathrm{CO}$ data located at $V_{\mathrm{LSR}}=-1.1 \pm 0.1$ $\mathrm{km} \mathrm{s}^{-1}$ that is well fit using a brightness temperature of $T_{\mathrm{mb}}=5.2 \pm 0.2 \quad \mathrm{~K}$ and an FWHM width of $\sigma_{\mathrm{FWHM}}=1.5 \pm 0.1 \mathrm{~km} \mathrm{~s}^{-1}$. The ${ }^{12} \mathrm{CO}$ isotopologue ${ }^{13} \mathrm{CO}$, also reveals line emission that is well fit to $T_{\mathrm{mb}} \sim 1.4 \pm 0.1 \quad \mathrm{~K} \quad$ at $\quad$ a velocity centroid of $V_{\mathrm{LSR}}=-0.9 \pm 0.1 \mathrm{~km} \mathrm{~s}^{-1}$ and an FWHM width of $\sigma_{\text {FWHM }}=1.0 \pm 0.1 \mathrm{~km} \mathrm{~s}^{-1}$, suggesting that the bulk of the mass is indeed at the farther distance. We then used a water maser cataloged with the HOP survey that is positionally coincident with IRAS 14482-5857, with a peak flux density of $5.1 \mathrm{Jy}$ at $-3.3 \mathrm{~km} \mathrm{~s}^{-1}$ with a line-width of $1.0 \mathrm{~km} \mathrm{~s}^{-1}$. We thus take the cloud to be at a velocity of $V_{\mathrm{LSR}}=-1.1 \mathrm{~km} \mathrm{~s}^{-1}$, which places it at a distance of $\sim 12.7 \mathrm{kpc}$.

5. Using the ${ }^{12} \mathrm{CO}$ and ${ }^{13} \mathrm{CO}$ data, we have used two methods for estimating the hydrogen column density and mass toward IRAS 14482-5857, which we find give average values of $N_{\mathrm{H}_{2}} \sim 1 \times 10^{21} \mathrm{~cm}^{-2}$ and $\sim 2 \times 10^{4}$ $M_{\odot}$.

6. Using the distance estimate obtained from the ${ }^{12} \mathrm{CO}$ data, we thus fit sizes for the strong radio continuum sources of $\lesssim 1 \mathrm{pc}$, strongly suggestive of UCHII regions. We then derive parameters for these sources: emission measures of $\sim 10^{6} \mathrm{pc} \mathrm{cm}^{-6}$, electron number densities $\left(\sim 10^{3} \mathrm{~cm}^{-3}\right)$, and rates of ionizing photon production of $\sim 10^{46-48} \mathrm{~s}^{-1}$, consistent with OB stars.

7. Three-color images of the region at $4.5,8$, and $24 \mu \mathrm{m}$ reveal a complex FIR spectro-morphology. The $4.5 \mu \mathrm{m}$ emission reveals a strong, compact source located at a minimum of the radio contours, indicative of a young stellar object. Emanating from the point source is what appears to be an outflowlike structure, which seems to align with the brightest of the three radio continuum peaks found in the object. The point source and outflowlike structure appears to be surrounded by a region of hot dust, as indicated by the abundance of $24 \mu \mathrm{m}$ emission found there. The emission within the diffuse $1-3 \mathrm{GHz}$ radio contours is dominated by $24 \mu \mathrm{m}$ emission, suggesting that the radio bubbles are "filled" with hot dust. Intersecting perpendicularly the lobular regions of hot dust is a region dominated by PAH emission, which also envelops the diffuse radio emission with many wispy segments.

8. Spectrally, the region as a whole is well fit by an optically thin thermal bremsstrahlung and two-component modified blackbody spectrum. The thermal bremsstrahlung component implies an emission measure of $\sim 1.4 \times 10^{5} \mathrm{pc} \mathrm{cm}^{-6}$, an electron density of $\sim 96 \mathrm{~cm}^{-3}$, a dust mass of $\sim 6.701 \times 10^{5} M_{\odot}$, and a production rate of ionizing photons of $N_{\mathrm{Ly}}$ of $\sim 7 \times 10^{47} \mathrm{~s}^{-1}$ for this source over its entire $3^{\prime} \times 4^{\prime}$ extent. The dust/FIR part of the spectrum is well fit by a two-component graybody with temperatures of $T_{\mathrm{d}}=19$ and $110 \mathrm{~K}$, respectively, strongly suggestive of massive star formation.

In totality, the evidence-distance estimates, strong radio sources that are consistent with $\mathrm{UCH}_{\text {II }}$ regions, the presence of a water maser, a high mass derived from the ${ }^{12} \mathrm{CO}$ observations, and a high dust temperature-strongly indicates that IRAS 14482-5857 is a site of massive star formation.

We thank the anonymous referee whose comments made the paper immeasurably better. D.I.J. thanks Petter Hofverberg for assistance with the observations and Ciriaco Goddi and Jürgen Ott for enlightening discussions and a critical reading of the manuscript. This research has made use of the SIMBAD database, operated at CDS, Strasbourg, France. The Australia Telescope is funded by the Commonwealth of Australia for operation as a National Facility managed by CSIRO.

\section{REFERENCES}

Bocchino, F., Parmar, A. N., Mereghetti, S., et al. 2001, A\&A, 367, 629 Bock, D. C.-J., Large, M. I., \& Sadler, E. M. 1999, AJ, 117, 1578 Bolatto, A. D., Wolfire, M., \& Leroy, A. K. 2013, ARA\&A, 51, 207

Breen, S. L., Caswell, J. L., Ellingsen, S. P., \& Phillips, C. J. 2010a, MNRAS, 406, 1487

Breen, S. L., Ellingsen, S. P., Caswell, J. L., \& Lewis, B. E. 2010b, MNRAS, 401, 2219

Breen, S. L., Ellingsen, S. P., Contreras, Y., et al. 2013, MNRAS, 435, 524

Burton, M. G., Braiding, C., Glueck, C., et al. 2013, PASA, 30, 44

Caswell, J. L. 1998, MNRAS, 297, 215

Churchwell, E., Babler, B. L., Meade, M. R., et al. 2009, PASP, 121, 213

Crocker, R. M., Jones, D. I., Melia, F., Ott, J., \& Protheroe, R. J. 2010, Natur, 463, 65

Culverhouse, T., Ade, P., Bock, J., et al. 2010, ApJ, 722, 1057

Culverhouse, T., Ade, P., Bock, J., et al. 2011, ApJS, 195, 8

Cyganowski, C. J., Whitney, B. A., Holden, E., et al. 2008, AJ, 136, 2391

Garay, G., Brooks, K. J., Mardones, D., Norris, R. P., \& Burton, M. G. 2002, ApJ, 579, 678

Goddi, C., Greenhill, L. J., Chandler, C. J., et al. 2009, ApJ, 698, 1165

Green, J. A., Caswell, J. L., Fuller, G. A., et al. 2012, MNRAS, 420, 3108

Hill, T., Burton, M. G., Minier, V., et al. 2005, MNRAS, 363, 405

Hindson, L., Thompson, M. A., Urquhart, J. S., et al. 2012, MNRAS, 421, 3418

Matthews, L. D., Greenhill, L. J., Goddi, C., et al. 2010, ApJ, 708, 80

Mauch, T., Murphy, T., Buttery, H. J., et al. 2003, MNRAS, 342, 1117

Maxted, N. I., Rowell, G. P., Dawson, B. R., et al. 2012, MNRAS, 422, 2230

Menten, K. M. 1991, ApJL, 380, L75

Molinari, S., Brand, J., Cesaroni, R., \& Palla, F. 1996, A\&A, 308, 573

Nicholas, B., Rowell, G., Burton, M. G., et al. 2011, MNRAS, 411, 1367

Ossenkopf, V., \& Henning, T. 1994, A\&A, 291, 943

Pineda, J. E., Caselli, P., \& Goodman, A. A. 2008, ApJ, 679, 481

Tielens, A. G. G. M. 2008, ARA\&A, 46, 289

Voronkov, M. A., Caswell, J. L., Ellingsen, S. P., Green, J. A., \& Breen, S. L. 2014, arXiv:1401.5179

Walsh, A. J., Hyland, A. R., Robinson, G., \& Burton, M. G. 1997, MNRAS, 291, 261

Walsh, A. J., Breen, S. L., Britton, T., et al. 2011, MNRAS, 416, 1764

Wood, D. O. S., \& Churchwell, E. 1989, ApJS, 69, 831

Wright, A. E., Griffith, M. R., Burke, B. F., \& Ekers, R. D. 1994, ApJS, 91, 111

Zinnecker, H., \& Yorke, H. W. 2007, ARA\&A, 45, 481 